\title{
Safe Needle Insertion Locations for Motor Point Injection of the Triceps Brachii Muscle: A Pilot Cadaveric and Ultrasonography Study
}

\author{
Hyun Jung Koo, MD ${ }^{1}$, Hye Jung Park, $\mathrm{MD}^{2}$, Geun-Young Park, MD, PhD ${ }^{3}$, \\ Yeonjae Han, $\mathrm{MD}^{3}$, Donggyun Sohn, $\mathrm{MD}^{3}$, Sun Im, MD, $\mathrm{PhD}^{3}$
}

\begin{abstract}
${ }^{1}$ Department of Rehabilitation Medicine, Seoul St. Mary's Hospital, College of Medicine, The Catholic University of Korea, Seoul; ${ }^{2}$ Department of Rehabilitation Medicine, National Traffic Injury Rehabilitation Hospital, Yangpyeong; ${ }^{3}$ Department of Rehabilitation Medicine, Bucheon St. Mary's Hospital, College of Medicine, The Catholic University of Korea, Bucheon, Korea
\end{abstract}

Objective To determine the location of the motor endplate zones (MoEPs) for the three heads of the triceps brachii muscles during cadaveric dissection and estimate the safe injection zone using ultrasonography.

Methods We studied 12 upper limbs of 6 fresh cadavers obtained from body donations to the medical school anatomy institution in Seoul, Korea. The locations of MoEPs were expressed as the percentage ratio of the vertical distance from the posterior acromion angle to the midpoint of the olecranon process. By using the same reference line as that used for cadaveric dissection, the safe injection zone away from the neurovascular bundle was identified in 6 healthy volunteers via ultrasonography. We identified the neurovascular bundle and its location with respect to the distal end of the humerus and measured its depth from the skin surface.

Results The MoEPs for the long, lateral, and medial heads were located at a median of $43.8 \%, 54.8 \%$, and $60.4 \%$ of the length of the reference line in cadaver dissection. The safe injection zone of the medial head MoEPs corresponded to a depth of approximately $3.5 \mathrm{~cm}$ from the skin surface and $1.4 \mathrm{~cm}$ away from the humerus, as determined by sonography.

Conclusion Correct identification of the motor points for each head of the triceps brachii would increase the precision and efficacy of motor point injections to manage elbow extensor spasticity.

Keywords Cadaver, Ultrasonography, Muscle spasticity, Botulinum toxins

Received March 28, 2019; Accepted July 30, 2019

Corresponding author: Sun Im

Department of Rehabilitation Medicine, Bucheon St. Mary's Hospital, College of Medicine, The Catholic University of Korea, 327 Sosa-ro, Bucheon 14647, Korea. Tel: +82-32-340-2170, Fax: +82-32-340-2173, E-mail: lafolia@catholic.ac.kr

ORCID: Hyun Jung Koo (http://orcid.org/0000-0002-5668-0821); Hye Jung Park (http://orcid.org/0000-0002-6731-4376); Geun-Young Park (http:// orcid.org/0000-0003-0084-2403); Yeonjae Han (http://orcid.org/0000-0002-0370-8747); Donggyun Sohn (http://orcid.org/0000-0003-3274-9182); Sun Im (http://orcid.org/0000-0001-8400-4911).

(c) This is an open-access article distributed under the terms of the Creative Commons Attribution Non-Commercial License (http://creativecommons.org/ licenses/by-nc/4.0) which permits unrestricted noncommercial use, distribution, and reproduction in any medium, provided the original work is properly cited. Copyright $\odot 2019$ by Korean Academy of Rehabilitation Medicine 


\section{INTRODUCTION}

Spasticity of the upper limbs is observed in cases of upper motor neuron diseases, such as stroke, traumatic brain injury (TBI), and multiple sclerosis [1]. Spasticity is one of the most physically debilitating conditions that impedes recovery of motor function. It makes the patient vulnerable to other complications such as contractures, peripheral muscular changes, and joint deformities [1]. In addition, the presence of spasticity may lead to increased inflammation in the joint, acceleration of the degenerative process, or increased pain. Spasticity must be controlled as it could adversely affect activities of daily living and elevate the risk of developing a pressure ulcer $[2,3]$. Compared with elbow flexor spasticity, elbow extensor spasticity is less common [4]. However, when present, elbow extensor spasticity causes motor imbalance with the triceps, thus, overpowering the elbow flexors, and impeding volitional elbow flexion. Elbow extensor spasticity treatment leads to improvement in upper limb function and prevention of joint contracture in individuals with weak biceps brachii muscle contraction and resistance [5].

Together with pharmacological treatments and operations, botulinum toxin injection is widely used to control focal spasticity in upper limbs [6]. It is important to inject botulinum toxin at exact motor end plates to maximize its chemodenervation effect [7]. The efficacy of botulinum toxin injections to control spasticity was maximized by injection at a motor point, specifically where the zones of the intramuscular endings are reported to be most dense, known as the motor endplate zones (MoEPs) [811]. Triceps brachii muscles are especially associated with elbow extensor spasticity more than the anconeus muscle [12]. The triceps brachii muscles consist of three heads, and each has a different contributing role in upper arm extension. The function of long head includes not only extension of the elbow, but also extension and adduction of the shoulder. The lateral head is the strongest, but it is usually not recruited except in activity against resistance. Inadvertent block of the long head instead of the medial head could result in aggravated shoulder instability since the long head is known to stabilize the glenohumeral joint and prevent inferior displacement of the humeral head. Among the three heads, the medial head is the main elbow extensor [13]. The medial head is located deep below the other two heads, and identification by simple surface palpation can be difficult. Also, the medial head is in close proximity to the neurovascular bundles. It is crucial to know not only the location of the motor points of the triceps brachii, but also the location of the safe injection zone, especially for the deeply located medial head. Although the locations of motor points in relation to surface anatomic landmarks for most upper extremity muscles are known, there are only limited reports $[14,15]$ for the triceps brachii muscles.

This study aims to identify the precise locations of the intramuscular motor nerve endings for the three heads of the triceps brachii muscles, which control elbow extensor spasticity, through both cadaveric dissection and ultrasonography localization. In this study, we offer the precise location of these motor points in relation to surface anatomic landmarks and recommend the most ideal injection points of the triceps brachii muscles using botulinum toxin.

\section{MATERIALS AND METHODS}

\section{Cadaveric dissection}

We studied twelve upper limbs of 6 fresh cadavers (2 males, 4 females) from body donations to the medical school anatomy institution in Seoul, Korea. The protocols of this study were approved by the Institutional Review Board of College of Medicine, The Catholic University of Korea (No. 2014-004). The informed consent was waived. A midline incision was made from the acromion angle to the midpoint of olecranon process with anatomical prone positioning. To expose the triceps brachii muscles, the skin and subcutaneous tissue were removed. We dissected the intramuscular course of the radial nerve to each head and followed them to the limits of visibility, to the terminal ramifications. Branches of the radial nerve to the long head, lateral head and medial head were dissected to show the motor points.

For each head, we measured the motor entry point (MEP), defined as the point where the nerve first makes its entry into the muscle; the proximal limit point (PLP), defined as the most proximal point where the intramuscular endings are observed; and the distal limit point (DLP), defined as the most distal point where the intramuscular endings are located. The area where the intramuscular endings are most dense was defined as the 
MoEP, the midpoint between the PLP and DLP, as mentioned in previous studies. Accurate administration of botulinum toxin with respect to the MoEPs is crucial for the best efficacy in the treatment of spasticity [8-11].

Both longitudinal and transverse coordinates of these three points (MEP, PLP, and DLP) were measured with respect to the reference line. The longitudinal coordinates were expressed as the percentage length of the reference line connecting from the acromion angle to the olecranon process. The transverse coordinates were expressed as the horizontal distance between the reference line to the motor points, namely MEP, PLP and DLP. When the points were ulnar to the reference line, negative values were given for the transverse coordinates (Fig. 1). The distance from the vertical reference line to the motor point was recorded as an $\mathrm{x}$ value $(\mathrm{cm})$ and the distance from the acromion angle to the point where the perpendicular line crossed the vertical reference line was defined as a y value (\%). The location of MoEPs were presented as negative $\mathrm{x}$ values, which were positioned on the ulnar side of the reference line. The y values were relative values, which were calculated as percentages. All measurements were performed by the same researcher using

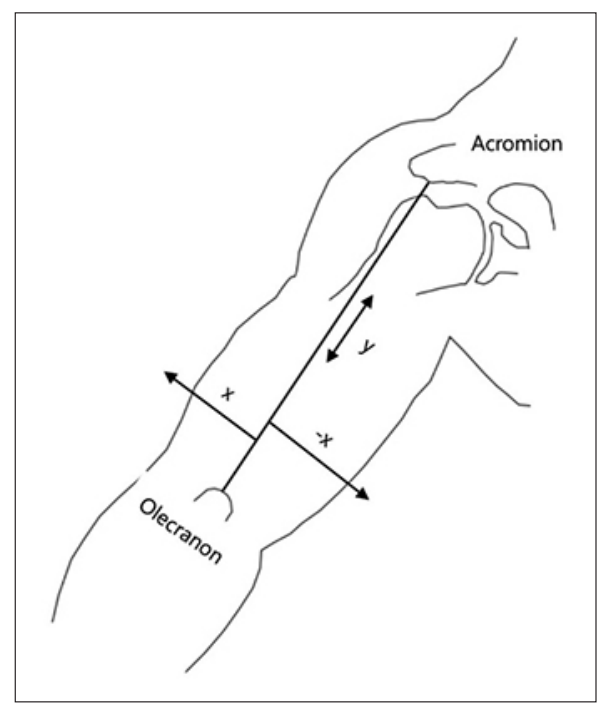

Fig. 1. Diagrammatic drawing of relationship between reference line and three points of the triceps muscle. The distance from the vertical reference line to each point was recorded as an $\mathrm{x}$ value $(\mathrm{cm})$ and the distance from the acromion angle to the point where perpendicular line crossed the vertical reference line was defined as a y value (\%). a digital caliper (Mitutoyo 500-180; Mitutoyo, Kanagawa, Japan).

Comparisons of the location of the three heads were performed with a Kruskal-Wallis test. For post-hoc tests, the Mann-Whitney U test was used. Data were expressed as the median and interquartile range (IQR). Statistical analysis was performed with SPSS version 18.0 (SPSS Inc., Chicago, IL, USA). For all statistical tests, a $\mathrm{p}=0.05$ level of significance was used.

\section{Ultrasonography study}

Ultrasonographic evaluations of 6 healthy volunteers (2 males, 4 females) were performed to evaluate the safe injection zone of the corresponding motor points retrieved from cadaveric dissection. With the subject in a sitting position and the elbow flexed to $90^{\circ}$ and the shoulder in a neutral position, the location of each MoEP of the three triceps brachii heads was marked on the skin along the same reference line as that used for the cadaveric dissection. The depth was defined as the distance between the skin surface at the MoEPs as confirmed from the cadaveric dissection and the central point of each muscle belly as observed on the transverse view ultrasonography image (HD11XE; Philips Healthcare, Andover, MA, USA) using a 5-12 MHz linear transducer. Administration of the injection at the central point of these MoEP locations would ensure that the injections are targeted to the intended muscle away from the nearby muscle fascia and prevent the inadvertent spread to the other muscles. After identifying the three triceps heads in transverse view at these MoEPs, we identified the neurovascular bundle and its location in relation to the border of the humerus and measured its depth from the skin surface. Depths at the same site were measured three times and the median value was used. Comparisons of the depths of the three heads were performed with a Kruskal-Wallis test.

\section{RESULTS}

\section{Cadaveric dissection results}

The median of total length of the reference line was $34.8 \mathrm{~cm}$. The median and IQR values of the longitudinal distance of the MEP, PLP, DLP and MoEP for each head are presented in Table 1. The MoEPs for the long, lateral, and medial heads were located at $43.8 \%, 54.8 \%$, and $60.4 \%$ distance of the reference line, respectively (Fig. 2). 
Table 1. Longitudinal coordinates of motor points of the triceps brachii muscle

\begin{tabular}{lcccr}
\hline & MEP & PLP & MoEP & \multicolumn{1}{c}{ DLP } \\
\hline Long head (\%) & $27.5 \pm 0.9^{*}(23.3-28.4)$ & $33.8 \pm 1.1^{*}(29.9-35.4)$ & $43.8 \pm 1.3^{*}(40.6-46.8)$ & $53.1 \pm 2.0^{*}(49.5-61.9)$ \\
Lateral head (\%) & $36.1 \pm 2.0^{*}(31.4-40.5)$ & $41.8 \pm 2.1^{*}(39.0-45.7)$ & $54.8 \pm 1.4^{*}(53.1-59.9)$ & $69.1 \pm 1.4(66.5-74.9)$ \\
Medial head (\%) & $48.7 \pm 1.5^{*}(46.4-50.8)$ & $53.9 \pm 0.9^{*}(52.2-57.3)$ & $60.4 \pm 1.1^{*}(57.5-63.7)$ & $66.9 \pm 1.8(61.4-72.4)$ \\
\hline
\end{tabular}

Values are presented as percentage ratio of the vertical distance from the acromion angle to the olecranon process. MEP, motor entry point; PLP, proximal limit point; MoEPs, motor endplate zones; DLP, distal limit point. ${ }^{*} \mathrm{p}<0.05$, group comparisons among the three heads were performed with a Kruskal-Wallis test.
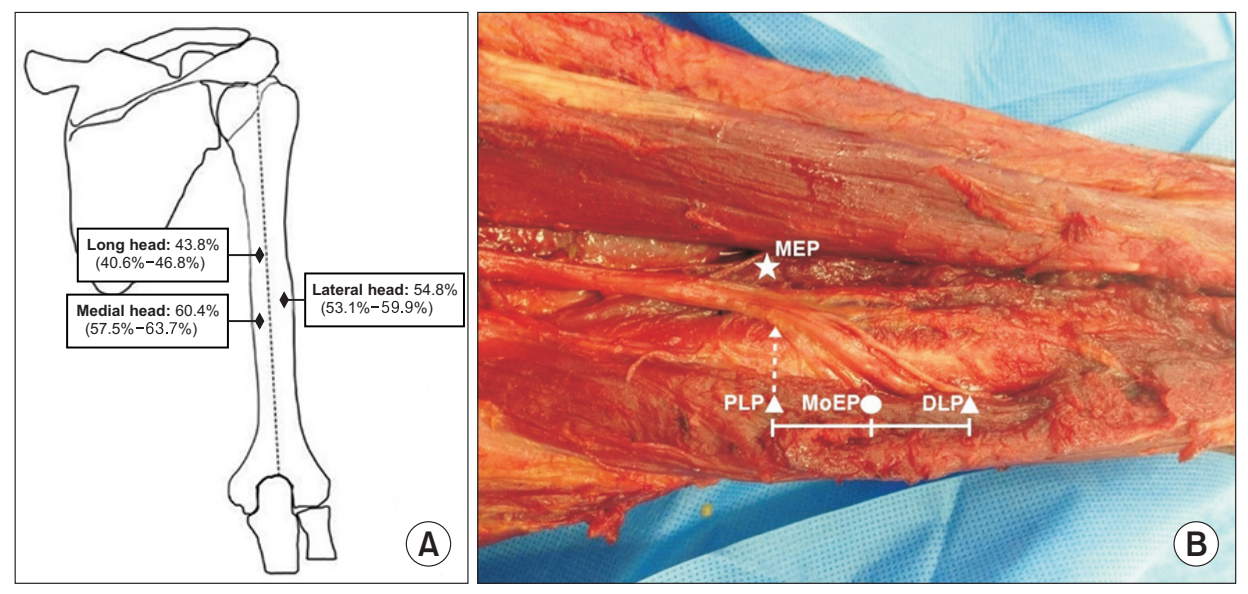

Fig. 2. Schematic drawing (A) of the location where the intramuscular endings are most densely located for each of the three heads of triceps brachii muscles and the branches of radial nerve in brachium innervating the triceps muscle (B). The branches of radial nerve at the motor entry point (MEP, star), proximal and distal limit point (PLP and DLP, white arrows) and where intramuscular nerve endings; the motor end plate zones (MoEP, circle) are most dense.

Table 2. Transverse coordinates of motor points of the triceps brachii muscle

\begin{tabular}{lcccc}
\hline & MEP & PLP & MoEP & DLP \\
\hline Long head $(\mathrm{cm})$ & $-3.7 \pm 0.5(-5.5-3.4)$ & $-4.0 \pm 0.4(-4.7-2.4)$ & $-4.0 \pm 0.3(-4.5-2.6)$ & $-4.0 \pm 0.3(-4.5-2.9)$ \\
Lateral head $(\mathrm{cm})$ & $3.3 \pm 0.5(2.4-3.6)$ & $3.3 \pm 0.3(2.7-4.5)$ & $3.3 \pm 0.2(2.7-4.2)$ & $3.2 \pm 0.3(2.7-3.6)$ \\
Medial head $(\mathrm{cm})$ & $-3.2 \pm 0.4(-5.0-2.5)$ & $-3.3 \pm 0.4(-5.0-2.5)$ & $-3.4 \pm 0.3(-4.4-2.5)$ & $-3.5 \pm 0.4(-4.0-2.4)$ \\
\hline
\end{tabular}

Values are presented as median \pm standard error (interquartile range). Negative values indicate position to the ulnar side of the reference line.

MEP, motor entry point; PLP, proximal limit point; MoEPs, motor endplate zones; DLP, distal limit point.

The median and IQR values of the transverse location for these points for each head is presented in Table 2. The MoEPs for the long, lateral, and medial heads were located at $-4.0,3.3,-3.4 \mathrm{~cm}$ from the reference line, respectively.

\section{Ultrasonography study}

The average body mass index of the healthy subjects was $21.22 \mathrm{~kg} / \mathrm{m}$. The long and lateral heads were located at a more superficial position than the medial head. The
MoEPs for the long, lateral, and medial heads were located at a median (IQR) depth of $2.0 \mathrm{~cm}(1.9-2.6 \mathrm{~cm}), 1.9$ $\mathrm{cm}(1.5-2.5 \mathrm{~cm})$, and $3.5 \mathrm{~cm}(3.0-3.8 \mathrm{~cm})$ from the skin surface, respectively. Although the depth distance of the long and lateral heads showed no statistical differences, the medial head was positioned at a statistically deeper level than the former two heads $(\mathrm{p}<0.05)$.

The neurovascular bundle was identified in close proximity to the medial head at a median (IQR) depth of 2.7 $\mathrm{cm}(2.5-3.1 \mathrm{~cm})$, with a distance of $1.0 \mathrm{~cm}(0.7-1.1 \mathrm{~cm})$ 

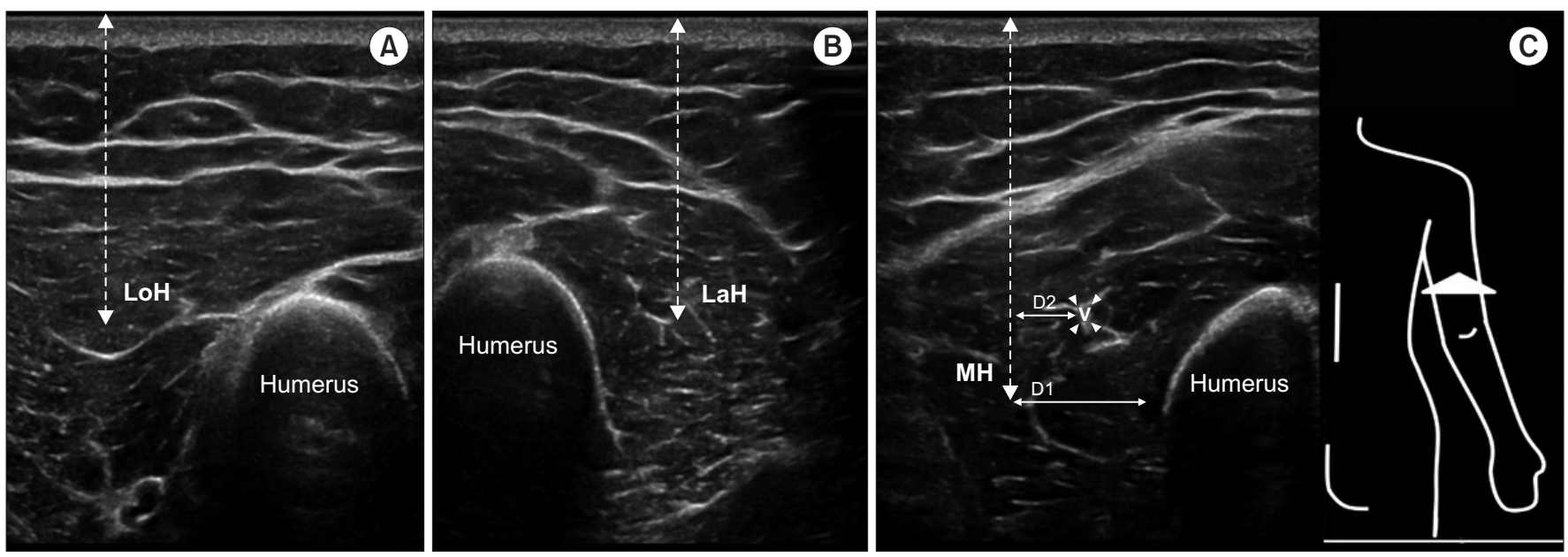

Fig. 3. Ultrasound scan, showing the MoEPs for the $\mathrm{LoH}(\mathrm{A})$, $\mathrm{LaH}(\mathrm{B})$, and $\mathrm{MH}(\mathrm{C})$ to be located at a median (interquartile range) depth of $2.0 \mathrm{~cm}(1.9-2.6 \mathrm{~cm}), 1.9 \mathrm{~cm}(1.5-2.5 \mathrm{~cm})$, and $3.5 \mathrm{~cm}(3.0-3.8 \mathrm{~cm})$ from the skin surface. The median distance between the central point of the muscle belly of the $\mathrm{MH}$ and the medial border of the humerus at the MoEP level (D1) was $1.4 \mathrm{~cm}(1.1-1.5 \mathrm{~cm})$. The median distance between the central point of the muscle belly of the MH and vessel at the MoEP level (D2) was $1.0 \mathrm{~cm}(0.7-1.1 \mathrm{~cm})$. MoEPs, motor endplate zones; LoH, long head; LaH, lateral head; $\mathrm{MH}$, medial head; $\mathrm{V}$, vessel (white arrow); D1, median distance between the central point of the muscle belly of the medial board and the medial border of the humerus; D2, median distance between the muscle belly of the MH and vessel.

from the central point of the muscle belly of the medial head. The median distance between the central point of the muscle belly of the medial head and the medial border of the humerus at the MoEP level was $1.4 \mathrm{~cm}$ (1.1-1.5 cm) (Fig. 3).

\section{DISCUSSION}

In this study, we report the results obtained from both the cadaveric and ultrasonography measurements on the precise location and depth of the motor points for selective block of the triceps brachii muscles. To selectively block the medial head, one should aim to target the motor points located at a distance of $60 \%$ of the length of the vertical reference line from the acromion angle and $-3.4 \mathrm{~cm}$ perpendicular from the vertical reference line. By marking the point on the skin as retrieved from the cadaveric dissection, sonography results revealed the safe injection zone would be at a depth of approximately 3.5 $\mathrm{cm}$ and approximately $1.4 \mathrm{~cm}$ away from the distal end of the humerus. Since these points were only $1 \mathrm{~cm}$ away from the neurovascular bundle, careful avoidance should be warranted. Unguided needle insertion to the deep-lying muscles solely based on surface anatomic landmarks may lead to unintentional injuries to the neurovascular bundles. Inadvertent puncture of the neurovascular bundles, especially in patients taking antiplatelet or anticoagulation medication, may lead to hematoma formation and even compartment syndrome. The depth distance data provided from this study may help guide clinicians to perform selective motor point injections to the triceps brachii with more precision, especially in the deep lying medial head, while avoiding unintended toxin leakage to the more superficial heads [16]. Current consensus recommends that, in order to achieve maximal effects of botulinum toxin injections, supplementary use of electrical stimulation or ultrasound guidance are recommended [17]. Sonography allows real-time visualization and easy accessibility, and it is painless and cost-effective [17]. Motor innervation of the radial nerve to the triceps brachii muscles had been identified for the long, lateral, and medial heads $[14,15]$ but with some discrepancies to the results provided in this study. Discrepancies could be due to different definitions of the reference line and anthropometric conditions. Also, although intramuscular endings are identified through cadaveric dissection, special staining techniques were not employed in our study. However, the midpoints between the most proximal and distal nerve endings, as used in this study, are an accurate estimation of the end points and have been consis- 
tently used to define the MoEP for spasticity treatment in many muscles [9-11].

Some limiting factors in this study need to be considered. Firstly, muscle depth was not estimated by cadaver dissection. As the duration of time after death increases, cadaveric tissue begins to lose viscoelasticity and consistency [18], making it inadequate for depth assessment. Although fresh cadavers were used, muscle depth is inherently different from that of living human skin. We tried to minimize the limitations of cadaveric study by adding ultrasonography of healthy individuals of similar age and sex to our study. Secondly, as has been stated from a previous study by Rha et al. [19], the safety window of the triceps muscles may be affected by the anthropometric characteristics of the subjects, such as body height and weight, and these should be assessed in future studies with a larger number of healthy volunteers. Whether the depth of the deep lying triceps muscles can be affected by the body habitus and arm circumference of a subject is an important point that should be pursued in future studies with more healthy subjects. Finally, the accuracy and safety of placing the injections at these locations to block the triceps muscles may need to be validated again in future cadaveric studies, where blind injections are placed first and then correct placement confirmed through cadaver dissection [20].

Despite these limiting factors, reports on the effect of botulinum toxin injections to the elbow extensors are scant. Though known to be less common, elbow extensor spasticity is nonetheless reported to develop after spasticity develops in the elbow flexor followed by the wrist flexors [21]. The parameters provided in this study have not been reported from previous cadaveric dissection studies and would be clinically relevant to physiatrists dedicated to the treatment of upper arm spasticity.

\section{CONFLICT OF INTEREST}

No potential conflict of interest relevant to this article was reported.

\section{AUTHOR CONTRIBUTION}

Conceptualization: Koo HJ, Park HJ, Im S. Methodology: Koo HJ, Park HJ, Im S, Park GY, Han YJ, Sohn DG. Funding acquisition: Koo HJ, Park HJ, Im S. Project administra- tion: Koo HJ, Im S. Visualization: Koo HJ, Im S, Park GY, Han YJ, Sohn DG. Writing - original draft: Koo HJ, Park HJ, Im S. Writing - review and editing: Koo HJ, Im S, Park GY, Han YJ, Sohn DG. Approval of final manuscript: all authors

\section{REFERENCES}

1. Ward AB. Spasticity treatment with botulinum toxins. J Neural Transm (Vienna) 2008;115:607-16.

2. Bai YL, Hu YS, Wu Y, Zhu YL, Zhang B, Jiang CY, et al. Long-term three-stage rehabilitation intervention alleviates spasticity of the elbows, fingers, and plantar flexors and improves activities of daily living in ischemic stroke patients: a randomized, controlled trial. Neuroreport 2014;25:998-1005.

3. Atiyeh BS, Hayek SN. Pressure sores with associated spasticity: a clinical challenge. Int Wound J 2005;2:7780.

4. Hefter H, Jost WH, Reissig A, Zakine B, Bakheit AM, Wissel J. Classification of posture in poststroke upper limb spasticity: a potential decision tool for botulinum toxin A treatment? Int J Rehabil Res 2012;35:22733.

5. Hierner R, Rollnik JD, Berger AC, Dengler R. Botulinum toxin type a for the treatment of biceps/triceps co-contraction in obstetrical brachial plexus lesions. Eur J Plast Surg 2001;24:2-6.

6. Thibaut A, Chatelle C, Ziegler E, Bruno MA, Laureys S, Gosseries O. Spasticity after stroke: physiology, assessment and treatment. Brain Inj 2013;27:1093-105.

7. Parratte B, Tatu L, Vuillier F, Diop M, Monnier G. Intramuscular distribution of nerves in the human triceps surae muscle: anatomical bases for treatment of spastic drop foot with botulinum toxin. Surg Radiol Anat 2002;24:91-6.

8. Shaari CM, Sanders I. Quantifying how location and dose of botulinum toxin injections affect muscle paralysis. Muscle Nerve 1993;16:964-9.

9. Van Campenhout A, Bar-On L, Desloovere K, Molenaers G. Role of motor end plate-targeted botulinum toxin type A injections in children with cerebral palsyitle. Acta Orthop Belg 2015;81:167-71.

10. Im S, Han SH, Choi JH, Lee JH, Ko YJ, Lee JI, et al. Anatomic localization of motor points for the neuromuscular blockade of hand intrinsic muscles involved in 
thumb-in-palm. Am J Phys Med Rehabil 2008;87:7039.

11. Alter KE, Karp BI. Ultrasound guidance for botulinum neurotoxin chemodenervation procedures. Toxins (Basel) 2017;10.

12. Brashear A, Elovic E. Spasticity: diagnosis and management. New York: Demos Medical Publishing; 2010.

13. Hamill J, Knutzen KM. Biomechanical basis of human movement. 3rd ed. Philadelphia: Lippincott Williams \& Wilkins; 2009.

14. Starkweather KJ, Gu Y, Gudas S, Murtaugh H, Frykberg B. PR_065: Localization of radial nerve motor points in the triceps muscle: a descriptive study. Arch Phys Med Rehabil 2006;87:e16.

15. Cho H, Lee HY, Gil YC, Choi YR, Yang HJ. Topographical anatomy of the radial nerve and its muscular branches related to surface landmarks. Clin Anat 2013;26:862-9.

16. Mayer NH, Whyte J, Wannstedt G, Ellis CA. Comparative impact of 2 botulinum toxin injection techniques for elbow flexor hypertonia. Arch Phys Med Rehabil
2008;89:982-7.

17. Berweck S, Schroeder AS, Fietzek UM, Heinen F. Sonography-guided injection of botulinum toxin in children with cerebral palsy. Lancet 2004;363:249-50.

18. Seok J, Oh CT, Kwon HJ, Kwon TR, Choi EJ, Choi SY, et al. Investigating skin penetration depth and shape following needle-free injection at different pressures: a cadaveric study. Lasers Surg Med 2016;48:624-8.

19. Rha DW, Im SH, Lee SC, Kim SK. Needle insertion into the tibialis posterior: ultrasonographic evaluation of an anterior approach. Arch Phys Med Rehabil 2010;91:283-7.

20. Rha DW, Han SH, Kim HJ, Won SY, Lee SC. Ultrasound-guided lateral approach for needle insertion into the subscapularis for treatment of spasticity. Arch Phys Med Rehabil 2012;93:1147-52.

21. Opheim A, Danielsson A, Alt Murphy M, Persson HC, Sunnerhagen KS. Upper-limb spasticity during the first year after stroke: stroke arm longitudinal study at the University of Gothenburg. Am J Phys Med Rehabil 2014;93:884-96. 